\title{
Towards understanding abscisic acid-mediated leaf senescence
}

\author{
LIANG Chengzhen \& CHU Chengcai* \\ State Key Laboratory of Plant Genomics and National Center for Plant Gene Research (Beijing), Institute of Genetics and Developmental \\ Biology, Chinese Academy of Sciences, Beijing 100101, China \\ Received January 28, 2015; accepted March 13, 2015; published online April 7, 2015 \\ Citation: Liang C, Chu C. Towards understanding abscisic acid-mediated leaf senescence. Sci China Life Sci, 2015, 58: 506-508, doi: \\ $10.1007 / \mathrm{s} 11427-015-4846-\mathrm{z}$
}

Senescence is a necessary part of most complex organisms that controlled by many complicated genetic programs. However, unlike animals and humans, leaf senescence has a great impact on nutrient recycling from source to sink to promote reproductive success, thus has strong adaptive advantages in plants $[1,2]$. Therefore, from the agricultural point of view, understanding the process of leaf senescence is extremely important for the breeding of higher-yielding crop with optimized nutritional qualities.

Onset and progression of leaf senescence is controlled primarily by developmental age, and it is also influenced by a number of endogenous and external factors that are integrated into the developmental age. Abscisic acid (ABA), a plant hormone identified in the 1960s [3], has been regarded as an important regulator in promoting leaf senescence. During leaf senescence, a dramatic increase in endogenous ABA levels is found in many plants, concomitantly with upregulation of a subset of ABA signaling genes and senescence-associated genes (SAGs), and exogenously applied ABA also induces expression of several SAGs which accelerate leaf senescence. In addition, a variety of biotic and abiotic stresses also elevate the ABA level and activate signaling pathways leading to senescence. These indicate that ABA plays a key role in regulating initiation and progression of leaf senescence; however, molecular basis of ABA-mediated leaf senescence signaling pathway is still largely unknown. Very recently, several components in two

*Corresponding author (email: ccchu@genetics.ac.cn) model plant species Arabidopsis and rice were identified, providing mechanistic insights into ABA-mediated leaf senescence signaling [2,4-6].

Receptor protein kinase 1 (RPK1) is an age-dependent action of an ABA-inducible receptor kinase, and acts as a positive regulator of leaf senescence in Arabidopsis [4]. $R P K 1$ encodes a membrane-bound protein that contains a leucine-rich repeat domain at its $\mathrm{N}$-terminus. Conditional overexpression of $R P K I$ at the mature stage significantly promoted leaf senescence, whereas RPKl knockout mutant exhibited reduced sensitivity to ABA-induced senescence. Consistently, RPK1 is an upstream component of ABA signaling and its expression is increased in an ABA-dependent manner throughout the progression of leaf senescence. However, induction of RPK1 expression in young plants leads to retarded growth but does not trigger the senescence symptoms, suggesting that function of RPK1 in ABAinduced leaf senescence is dependent on the developmental age.

Senescence-associated gene113 (SAG113) is a negative regulator of ABA signaling controlling water loss during senescence in Arabidopsis [5]. SAG113 encodes a Golgi-localized $\mathrm{PP} 2 \mathrm{C}$ family protein phosphatase, which is able to complement the yeast PP2C-deficient ptdl mutant, confirming that SAG113 is the functional ortholog of PTC1. SAG113 is induced by ABA in senescing leaves and its expression is significantly repressed in both ABA biosynthesis and signaling mutant aba2-1 and abi4-1. The loss-offunction mutants of SAG113 display a delay in leaf senes- 
cence, the stomata movement is also more sensitive to ABA, and the water loss rate is significantly reduced. In contrast, inducible overexpression of $S A G 113$ causes a precocious leaf senescence symptom, less sensitive to ABA treatment in stomata movement, and faster water loss. These suggest that SAG113 positively regulates ABA-induced leaf senescence via inhibition of stomata closure, finally accelerates water loss in senescing leaves.

NAP (NAC-like, activated by apetala3/pistillata) was reported as an important positive regulator that controls leaf senescence in both Arabidopsis and rice by four independent groups [2,6-8]. It encodes plant specific NAC (NAM, ATAF1/2, CUC2) transcription activator. Overexpression of either AtNAP or OSNAP could trigger precocious agedependent and dark-induced leaf senescence, and knockdown of it significantly delayed the senescence both in Arabidopsis and rice. Interestingly, AtNAP and OsNAP are specifically induced by exogenous ABA, whereas OSNAP expression is repressed in both abal and $a b a 2$, two ABA biosynthetic mutants, thus implying a possible functional similarity between AtNAP and OsNAP as positive mediators of ABA-signaling and leaf-senescence processes in plants.

Although both AtNAP and OsNAP positively regulate ABA-mediated leaf senescence, it shows different mechanisms in these two species. In Arabidopsis, AtNAP regulates leaf senescence processes via directly binding to a 9-bp core sequence (CACGTAAGT) of the SAG113 promoter to form an ABA-AtNAP-SAG113 PP2C regulatory chain for controlling dehydration in senescing leaves [9]. However, in rice, OsNAP fine-tunes the leaf senescence by directly regulating the expression of SAGs including chlorophyll degradation-related genes and nutrient transportrelated genes in an age-dependent manner [2]. This difference implied that AtNAP and OsNAP may have distinctive substrate specificities in Arabidopsis and rice. Intriguingly, in rice, high OsNAP expression levels could repress ABA biosynthesis via a feedback mechanism in developmental senescence process [2]; however, which knockout of AtNAP in Arabidopsis results in a dramatic decrease in ABA content through binding to the promoter of abscisic aldehyde oxidase 3 (AAO3, encoding the enzyme of catalyzing the last step of ABA biosynthesis) and transactivates its expression during dark-induced senescence [6]. This discrepancy between rice and Arabidopsis may be attributed to different mechanisms of NAP in regulating developmental senescence in rice and dark-induced senescence in Arabidopsis. Comparative transcriptome analysis revealed that there do exist the differences in gene expression and hormone signaling pathways between developmental senescence process and dark-induced leaf senescence [10]. To this end, a detailed analysis of ABA content alterations in dark-induced senescence of AtNAP overexpression lines as well as in developmental senescence process of atnap mutant and
AtNAP overexpression lines is imperative. Alternatively, this discrepancy also might be due to the different species between dicots and monocots. Nevertheless, given that AtNAP/OsNAP is a functional ABA-mediated senescence signaling component, these studies highlight that NAP serves as an important link between ABA and leaf senescence in higher plants.

Despite the significant progress recently in elucidating molecular mechanism of ABA-mediated leaf senescence, it is still at the beginning towards a full understanding of the molecular networks of this complex biological process. Since it is difficult to identify the genes of ABA-regulated leaf senescence by forward genetic approach because of functional redundancy, the identification of new components controlling ABA-induced leaf senescence needs to be accomplished by different approaches. A number of recent studies demonstrated that systems biology approaches, especially by combining the different omics approaches, should facilitate the identification of new components underlying ABA-mediated leaf senescence and construction of its complete network. On the other hand, the analysis of natural variations during evolution and selection, combined with genome-wide association study, should be an alternative way to identify important senescence-associated loci that are difficult to identify using genetic approaches.

$\mathrm{ABA}$ is also a plant stress hormone integrating various stress signals and controlling downstream stress responses. Leaf senescence indeed often occurs in mutants affected in synthesis or perception of ABA under certain stress conditions. So far we did not know anything about how ABA precisely balances its role in senescence and stress signaling during the development of plant. Our work gave a hint that pollination might play the crucial role in the crosstalk between ABA-induced and age-dependent senescence initiation [2].

1 Lim PO, Kim HJ, Nam HG. Leaf senescence. Annu Rev Plant Biol, 2007, 58: 115-136

2 Liang C, Wang Y, Zhu Y, Tang J, Hu B, Liu L, Ou S, Wu H, Sun X, Chu J, Chu C. OsNAP connects abscisic acid and leaf senescence by fine-tuning abscisic acid biosynthesis and directly targeting senescence-associated genes in rice. Proc Natl Acad Sci USA, 2014, 111: 10013-10018

3 Sheard LB, Zheng N. Signal advance for abscisic acid. Nature, 2009, 462: 575-576

4 Lee IC, Hong SW, Whang SS, Lim PO, Nam HG, Koo JC. Agedependent action of an ABA-inducible receptor kinase, RPK1, as a positive regulator of senescence in Arabidopsis leaves. Plant Cell Physiol, 2011, 52: 651-662

5 Zhang K, Xia X, Zhang Y, Gan SS. An ABA-regulated and Golgi-localized protein phosphatase controls water loss during leaf senescence in Arabidopsis. Plant J, 2012, 69: 667-678

6 Yang J, Worley E, Udvardi M. A NAP-AAO3 regulatory module promotes chlorophyll degradation via ABA biosynthesis in Arabidopsis leaves. Plant Cell, 2014, 26: 4862-4874

7 Guo Y, Gan S. AtNAP, a NAC family transcription factor, has an 
important role in leaf senescence. Plant J, 2006, 46: 601-612

8 Zhou Y, Huang W, Liu L, Chen T, Zhou F, Lin Y. Identification and functional characterization of a rice NAC gene involved in the regulation of leaf senescence. BMC Plant Biol, 2013, 13: 132

9 Zhang K, Gan SS. An abscisic acid-AtNAP transcription factor-SAG113 protein phosphatase $2 \mathrm{C}$ regulatory chain for controlling dehydration in senescing Arabidopsis leaves. Plant Physiol, 2012,
158: 961-969

10 Buchanan-Wollaston V, Page T, Harrison E, Breeze E, Lim PO, Nam HG, Lin JF, Wu SH, Swidzinski J, Ishizaki K, Leaver CJ. Comparative transcriptome analysis reveals significant differences in gene expression and signalling pathways between developmental and dark/starvation-induced senescence in Arabidopsis. Plant J, 2005, 42: $567-585$

Open Access This article is distributed under the terms of the Creative Commons Attribution License which permits any use, distribution, and reproduction in any medium, provided the original author(s) and source are credited. 